\title{
Systolic and Diastolic Ventricular Function Assessed by Pressure-Volume Loops in the Stage 21 Venous Clipped Chick Embryo
}

\author{
SANDRA STEKELENBURG-DE VOS, PAUL STEENDIJK, NICOLETTE T.C. URSEM, \\ JURIY W. WLADIMIROFF, RENÉ DELFOS, AND ROBERT E. POELMANN \\ Department of Obstetrics and Gynecology [S.S.-D., N.T.C.U., J.W.W.], Erasmus MC, 3015 GD Rotterdam, \\ The Netherlands, Department of Cardiology [P.S.], Department of Anatomy and Embryology [R.E.P.], \\ Leiden University Medical Center, 2300 RC Leiden, The Netherlands, J.M. Burgers Centre for Fluid \\ Dynamics [R.D.], Laboratory for Aero and Hydrodynamics, Delft University of Technology, 2628 CA \\ Delft, The Netherlands
}

\begin{abstract}
Cardiac pressure-volume relations enable quantification of intrinsic ventricular diastolic and systolic properties independent of loading conditions. The use of pressure-volume loop analysis in early stages of development could contribute to a better understanding of the relationship between hemodynamics and cardiac morphogenesis. The venous clip model is an intervention model for the chick embryo in which permanent obstruction of the right lateral vitelline vein temporarily reduces the mechanical load on the embryonic myocardium and induces a spectrum of outflow tract anomalies. We used pressure-volume loop analysis of the embryonic chick heart at stage 21 ( $3.5 \mathrm{~d}$ of incubation) to investigate whether the development of ventricular function is affected by venous clipping at stage 17 , compared with normal control embryos. Steady state hemodynamic parameters demonstrated no significant differences between the venous clipped and control embryos. However, analysis of pressure-volume relations showed a significantly lower end-systolic elastance in the clipped embryos (slope of the end-systolic pressure-volume relation: $5.68 \pm 0.85$ versus $11.76 \pm 2.70 \mathrm{~mm} \mathrm{Hg} / \mu \mathrm{L}, p<0.05$ ), indicating reduced contractility. Diastolic stiffness tended to be increased in the clipped embryos (slope of end-diastolic pressure-volume relation: $2.74 \pm 0.56$ versus $1.67 \pm 0.21, p=$ 0.103 ), but the difference did not reach statistical significance. The results of the pressure-volume loop analysis show that $1 \mathrm{~d}$ after venous obstruction, development of ventricular function is
\end{abstract}

\section{ABSTRACT}

affected, with reduced contractility. Pressure-volume analysis may be applied in the chick embryo and is a sensitive technique to detect subtle alterations in ventricular function. (Pediatr Res 57: 16-21, 2005)

CO, cardiac output
EDP, end-diastolic pressure
EDPVR, end-diastolic pressure-volume relation
EDV, end-diastolic volume
$\mathbf{E}_{\mathbf{E D}}$, end-diastolic stiffness
$\mathbf{E}_{\mathbf{E S}}$, end-systolic elastance
EF, ejection fraction
ESP, end-systolic pressure
ESPVR, end-systolic pressure-volume relation
ESV, end-systolic volume
ESV, end-systolic volume at 2 mm Hg
HH, Hamburger and Hamilton
HR, heart rate
PER, peak ejection rate
PFR, peak filling rate
PHT, pressure half time
SV, stroke volume
SW, stroke work

EDP, end-diastolic pressure

EDPVR, end-diastolic pressure-volume relation

EDV, end-diastolic volume

$\mathbf{E}_{\mathbf{E D}}$, end-diastolic stiffness

$\mathbf{E}_{\mathbf{E S}}$, end-systolic elastance

EF, ejection fraction

ESV, end-systolic volume

HR, heart rate

PER, peak ejection rate

SW, stroke work
Animal models are required to study mechanisms of early cardiovascular development. The chick embryo has been used as a model for many decades because the embryonic chick heart resembles the developing human heart in many aspects $(1,2)$. The

Received June 17, 2004; accepted July 19, 2004

Correspondence: N.T.C. Ursem, Ph.D., Department of Obstetrics and Gynecology, Erasmus MC - University Medical Center Rotterdam, Dr. Molewaterplein 40, 3015 GD Rotterdam, The Netherlands; e-mail: n.ursem@erasmusmc.nl

Supported by Grant 2000.016 from the Netherlands Heart Foundation.

DOI: 10.1203/01.PDR.0000147734.53277.75 intricate relationship between hemodynamics and cardiac morphogenesis has been a major study topic in this model.

The venous clip model is an intervention model for the chick embryo in which permanent obstruction of the right lateral vitelline vein temporarily reduces the mechanical load on the embryonic myocardium and induces a spectrum of outflow tract anomalies (3). This model was designed to obtain insight into the effects of altered venous return patterns on cardiac morphogenesis and malformations (4). 
In the chick embryo, information on cardiac function can be assessed through blood flow velocities and pressure wave form recordings (1,5-7). In a previous study using a $20 \mathrm{MHz}$ Doppler velocity meter, we have demonstrated that the experimental alteration of venous return caused by venous clipping has major acute effects on hemodynamics in the HH stage 17 chick embryo $(3,8)$. Peak systolic velocity, peak blood flow, and SV are all significantly decreased for up to $5 \mathrm{~h}$ after clipping. However, dorsal aortic Doppler measurements give indirect information on cardiac function. Direct information can be assessed using cardiac pressure-volume loop analysis (9). This method enables assessment of ventricular diastolic and systolic properties independent of loading conditions. Pressure-volume analysis has been widely used in both human and animal studies (10-12). Only a few studies, in (near-term) fetal lambs and chick embryos, have been done during embryonic or fetal development $(9,13-15)$.

The use of pressure-volume loop analysis in early stages of development could contribute to a better understanding of cardiovascular developmental processes and especially the relationship between cardiac function and cardiac morphogenesis, as cardiac morphogenesis depends on the interaction between genetics and epigenetic influences like fluid mechanical forces (16).

Because mechanical load influences cardiac development and clipping the right lateral vitelline vein alters mechanical load, we tested the hypothesis that clipping could lead to long-term changes in ventricular function. For this purpose a custom-made workstation was used to simultaneously measure intraventricular pressure and capture video images of the beating ventricle. We performed pressure-volume loop analysis of the embryonic chick heart at stage 21 to investigate whether the development of ventricular function is affected by venous clipping at stage 17 compared with normal control embryos.

\section{MATERIALS AND METHODS}

Animals. Fertilized white Leghorn chick eggs (Gallus gallus (L.)) were obtained from Charles River Laboratories (Extertal, Germany) and were incubated at $37-38^{\circ} \mathrm{C}$, with the blunt end up and at a relative humidity of $70-80 \%$. The embryos were exposed by creating a window in the shell and removing the overlying membranes. Twenty-one embryos that were at $\mathrm{HH}$ stage 17 and that showed no bleeding or deformities were selected (8). The material was subdivided into venous clipped embryos $(n=11)$, in which the right vitelline vein was obstructed with an aluminium microclip, and control embryos $(n=10)$. A more detailed description of the clipping procedure has been published previously (3). During the experiments, the eggs were placed on a heating element at $37^{\circ} \mathrm{C}$. Experiments were approved by the Animal Experimentation Review Board of the Erasmus Medical Center and were performed in accordance with Dutch Law for animal experimentation

Intraventricular pressure. Blood pressure was measured in the ventricle at HH stage 21 (Fig. 1) using a servo-null system (model 900A, World Precision Instruments, Sarasota, FL) and a fluid-filled $(2 \mathrm{M} \mathrm{NaCl}) 5-10 \mu \mathrm{m}$ glass micropipette attached to a microelectrode. Intraventricular pressure was calculated as the difference between the measured pressure and the pressure recorded when the tip of the pipette was placed in the extraembryonic fluid adjacent to the ventricular puncture site. Pressure data were sampled at $50 \mathrm{~Hz}$ and stored on a personal computer. The working principle of this pressure transducer is described in detail by Heineman and Grayson (17). The dynamic response of the system was tested over the frequency range of $0.5-20 \mathrm{~Hz}$ (as this frequency range is relevant to chicken embryonic heart rate) by applying air pressure fluctuations on top of a liquid bath in which the probe with

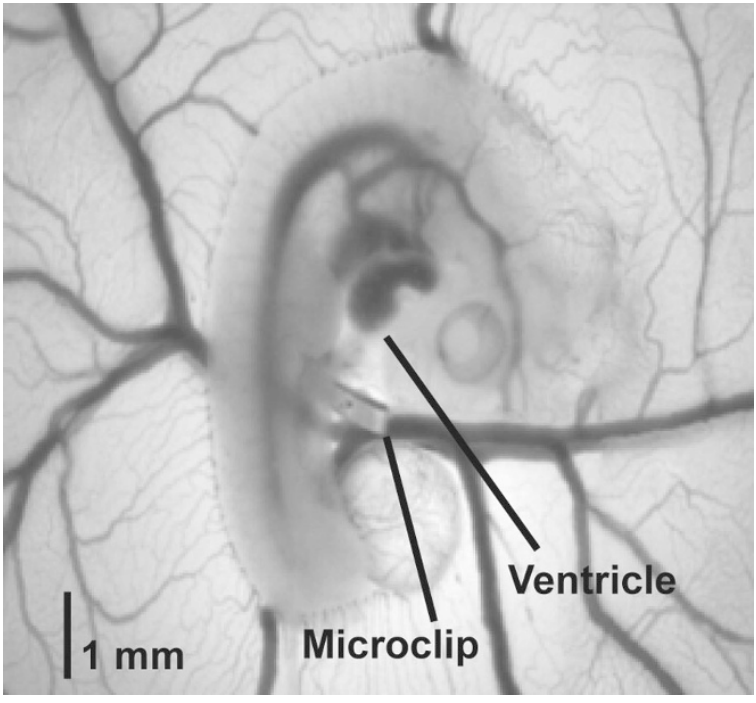

Figure 1. Stage 21 chick embryo ( $3.5 \mathrm{~d}$ of incubation).

micropipette was inserted. The actual pressure was measured simultaneously, using a pressure transducer of known flat response up to $130 \mathrm{~Hz}$ (Setra Systems, Boxborough, MA). The dynamic response of the servo-null system relative to the actual pressure is shown in Figure 2. We conclude that at the HR frequency $(2-3 \mathrm{~Hz})$ the signal amplitude is amplified (underdamped) by about $1 \%$ only and the phase delay is about 5-6 degrees, or $7 \mathrm{~ms}$, or about one-quarter of the frame time interval. For the fourth harmonic $(10 \mathrm{~Hz})$ (at four times the HR frequency), where still $2-3 \%$ of the signal energy is present in the energy spectrum, the signal amplitude is amplified by about $20 \%$ and the phase delay is 25-30 degrees, or $8 \mathrm{~ms}$, or about one-quarter of the frame time interval. We therefore conclude that the pressure is measured with sufficient accuracy.

Furthermore, to avoid aliasing of higher frequencies into the low-frequency domain, pressure data were filtered with a second-order low-pass Butterworth filter (Krohn-Hite Corporation, Brockton, MA) set at the sampling frequency.

Intraventricular volume. During the pressure measurements, the ventricle was visualized using video imaging with a stereo microscope (model SV 6, Carl Zeiss, Oberkochen, Germany) and a video camera (model SSC-M370CE, Sony Corporation, Tokyo, Japan). The video images were acquired simultaneously with the analog pressure signal at $50 \mathrm{~Hz}$ and stored on a personal computer. Epicardial surface area was calculated from magnified video images displaying the ventricle using a custom-built analysis program (IMAQ Vision, National Instruments, Austin, TX). Ventricular volume (V) was derived from epicardial area (A) using a simplified ellipsoid geometric model: $\mathrm{V}=0.65 \mathrm{~A}^{3 / 2}$, as described by Keller et al. (9).

For each clipped and control embryo, two to four consecutive (baseline) cardiac cycles were analyzed. Furthermore, to evaluate ventricular response to reduced venous return, a fourth-order vitelline vein was incised to produce

A
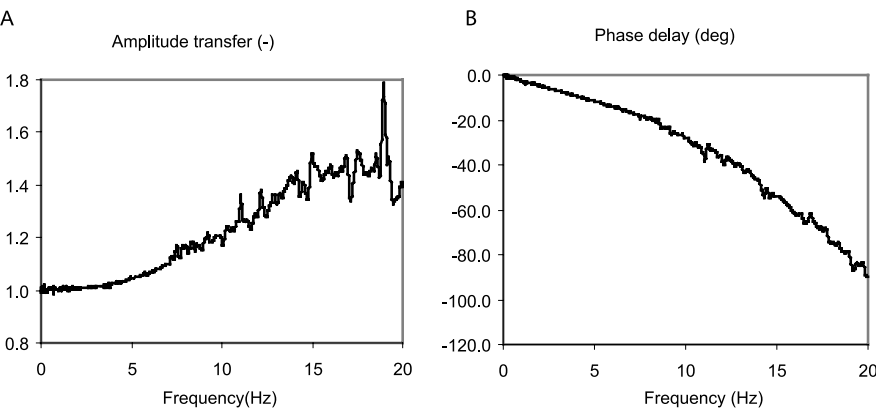

Figure 2. Dynamic frequency response of the servo-null system. $(A)$ The amplitude transfer curve of the measured pressure signal over a frequency range of $0.5-20 \mathrm{~Hz}$ relative to the actual pressure. $(B)$ The phase delay curve of the signal. 
venous hemorrhage, which results in acute preload reduction. The cardiac cycles following hemorrhage were also recorded and subsequently analyzed to derive ventricular pressure-volume relations. By the latter procedure information can be obtained about ventricular contractility (through the end-systolic pressure volume relation) and $\mathrm{E}_{\mathrm{ED}}$ (through the end-diastolic pressure volume relation).

Finally, maximum contraction of the heart with $2 \mathrm{M} \mathrm{NaCl}$ was induced for determination of ventricular wall volume by video imaging. The solution (1-3 $\mu \mathrm{L})$ was applied directly on the ventricle. Cavity volume was calculated as total volume minus wall volume. We validated this method by a separate study in a set of four embryos at stage 21 in which wall volume obtained by video imaging was compared with wall volume obtained by the counting method of Cavalieri, detailed by Gundersen and Jensen (18). The wall volume of the ventricles was determined after $2 \mathrm{M} \mathrm{NaCl}$ application by video imaging, directly followed by fixation with $4 \%$ paraformaldehyde in $0.1 \mathrm{M}$ phosphate buffer. The hearts were embedded in paraffin, serially sectioned at $5-\mu \mathrm{m}$ thickness, and stained with hematoxylin-eosin for stereologic examination. The number of points on a grid hitting 15 sections of the ventricle was counted. The first section was taken randomly and the other sections were taken systematically. From the counted numbers, the volumes could be calculated by Cavalieri's formula: $\mathrm{V}=\Sigma \mathrm{P} \cdot \mathrm{M}^{-2} \cdot \mathrm{a} \cdot \mathrm{d}$, where $\mathrm{V}$ is the volume in cubic millimeters, $\Sigma \mathrm{P}$ is the total number of counted points on the 15 sections, $\mathrm{M}$ the magnification, a is the point area in square millimeters, and $\mathrm{d}$ is the distance between the counted sections in millimeters.

The mean ventricular wall volume obtained by video imaging was $0.171 \pm$ $0.009 \mu \mathrm{L}$ and mean myocardial wall volume obtained by Cavalieri's method was $0.157 \pm 0.010 \mu \mathrm{L}$, which means that ventricular wall volume is overestimated by $8.9 \%$. Therefore, we concluded that the video imaging procedure correlates well with stereologically obtained volumes and we chose not to correct for this overestimation in the calculations.

Calculations. All data were interpolated using a natural spline function (LabVIEW 6.0, National Instruments, Austin, TX). Data analysis and calculations were performed using custom-made software (Circlab, Leiden University Medical Center, Leiden, the Netherlands). For each variable the mean value of two to four consecutive cardiac cycles was calculated. Pressure and volume data were plotted as pressure-volume loops (Fig. 3). End-systole is represented by the upper-left corner of the pressure-volume loops and was defined as the moment in the cardiac cycle with the maximum instantaneous pressure to volume ratio or time-varying elastance $\mathrm{E}(\mathrm{t})=\mathrm{P}(\mathrm{t}) /[\mathrm{V}(\mathrm{t})-\mathrm{Vd}]$ (19). Vd represent volume at zero pressure and was determined using an iterative approach as described by Kono et al. (20).

The lower-right corner of the pressure-volume loop represents end-diastole as the onset of ventricular contraction and was defined as the point at which the first derivative of the pressure wave form rose sharply from baseline. Endsystolic and end-diastolic ventricular pressures and volumes (ESP, EDP, mm $\mathrm{Hg}$; ESV, EDV, $\mu \mathrm{L}$ ) were determined as the instantaneous values $\mathrm{P}(\mathrm{t})$ and, respectively, $\mathrm{V}(\mathrm{t})$ at these time-points. Heart rate $(\mathrm{HR}, \mathrm{bpm})$ was calculated from the beat-to-beat cycle length. SV $(\mu \mathrm{L})$ was calculated as the difference between EDV and ESV. Cardiac output $(\mathrm{CO}, \mu \mathrm{L} / \mathrm{min})$ was calculated as $\mathrm{HR}$ multiplied by SV. EF (\%) was determined by SV divided by EDV. SW $(\mu \mathrm{L} / \mathrm{mm} \mathrm{Hg})$ was calculated as the area of the pressure-volume loop. The
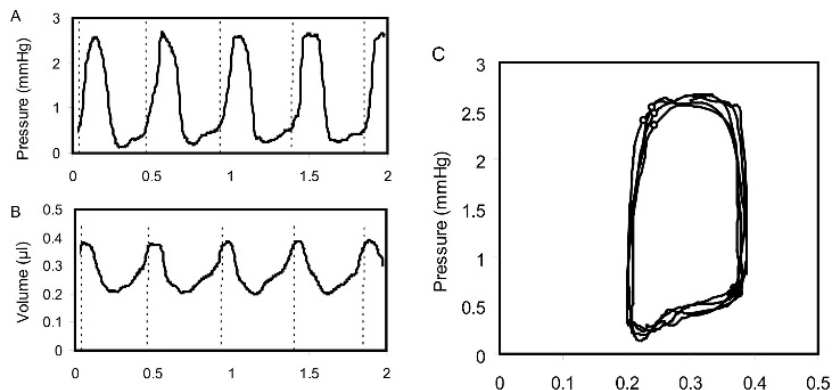

Figure 3. Representative stage 21 baseline pressure-volume loops $(C)$ built up from simultaneously acquired pressure $(A)$ and volume data $(B)$. Solid circles depict end-diastolic points and open circles depict end-systolic points (C). Also, end-diastolic points are represented by dotted lines in panels $A$ and $B$. maximum and minimum first derivatives of pressure were determined $(\mathrm{dP} /$ $\left.\mathrm{dt}_{\mathrm{MAX}}, \mathrm{dP} / \mathrm{dt}_{\mathrm{MIN}}, \mathrm{mm} \mathrm{Hg} / \mathrm{s}\right)$. PFR ( $\left.\mu \mathrm{L} / \mathrm{s}\right)$ and PER $(\mu \mathrm{L} / \mathrm{s})$ were identified as the maximum and minimum first derivatives of ventricular volume with respect to time $[\mathrm{dV}(\mathrm{t}) / \mathrm{dt}]$. The relaxation time constant Tau $(\tau, \mathrm{ms})$ was calculated as the time constant of monoexponential pressure decay during isovolumic relaxation. The isovolumic period was defined as the time period between the moment of $\mathrm{dP} / \mathrm{dt}_{\mathrm{MIN}}$ and the time point at which $\mathrm{dP}(\mathrm{t}) / \mathrm{dt}$ reached $10 \%$ of $\mathrm{dP} / \mathrm{dt}_{\mathrm{MIN}}$ (21). In addition, we calculated PHT (ms) as the time interval between the moment of $\mathrm{dP} / \mathrm{dt}_{\mathrm{MIN}}$ and the moment that pressure has dropped to $50 \%$ of the instantaneous pressure at the moment of $\mathrm{dP} / \mathrm{dt}_{\mathrm{MIN}}$.

In addition to these steady-state indices, we used the baseline pressurevolume loops combined with loops obtained after venous hemorrhage to determine systolic and diastolic pressure-volume relationships as loadindependent measures of systolic and diastolic ventricular function. Systolic function was characterized by $\mathrm{E}_{\mathrm{ES}}(\mathrm{mm} \mathrm{Hg} / \mu \mathrm{L})$, which is the linear slope of the ESPVR (Fig. 4). Also, the position of the ESPVR, defined as the volumeintercept at $2 \mathrm{~mm} \mathrm{Hg}\left(\mathrm{ESV}_{2}, \mu \mathrm{L}\right)$, was determined to quantify the volumeintercept at a physiologic pressure, rather than at pressure equals zero (22).

Diastolic ventricular function was determined by calculating $\mathrm{E}_{\mathrm{ED}}(\mathrm{mm}$ $\mathrm{Hg} / \mu \mathrm{L}$ ) as the slope of the EDPVR: EDP $=\mathrm{a}+\mathrm{E}_{\mathrm{ED}} \cdot \operatorname{EDV}(23)$.

Statistical analysis. Data are presented as mean \pm SEM. For comparison of the clipped embryos with control embryos, unpaired $t$ tests were performed using SPSS 10.1 software (SPSS Inc., Chicago, IL). When Levene's test showed inhomogeneity of variance or when the data were not normally distributed according to the Shapiro-Wilk test, a $\log (\ln )$ transformation was performed before testing group differences (24). Values of $p<0.05$ were considered statistically significant.

\section{RESULTS}

Steady state hemodynamic parameters were determined from baseline pressure and volume signals and corresponding pressure-volume loops as depicted in Figure 3. Results are summarized in Table 1 and do not reveal any significant difference between clipped and control embryos.

Figure 4 shows representative pressure-volume loops from a clipped and a control embryo. The gray lines represent loops at baseline, whereas the black lines are loops obtained after hemorrhage induced by incision of a fourth-order vitelline vein. End-systolic and end-diastolic pressure-volume points are marked and the resulting pressure-volume relations are indicated. Note that, compared with the control embryo, in the clipped embryo the ESPVR was less steep, indicating reduced
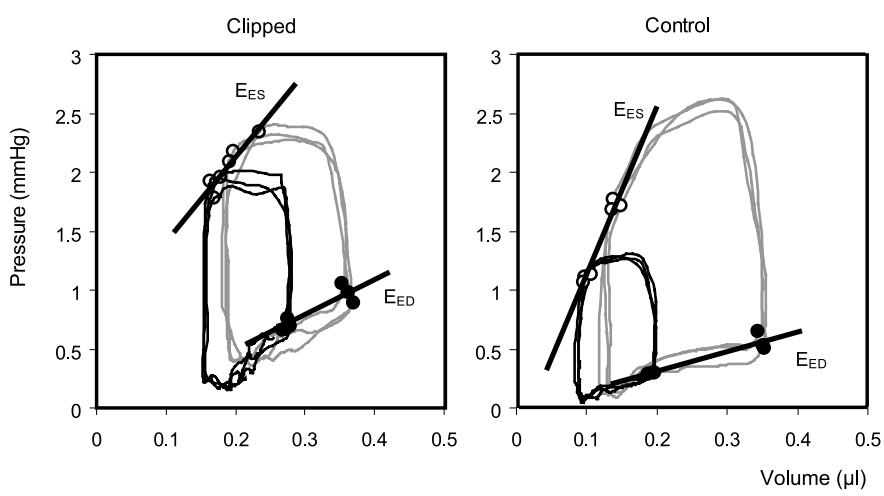

Figure 4. Representative pressure-volume loops of a clipped and a control embryo at stage 21. Both baseline-loops (gray lines) and loops after venous hemorrhage (black lines) are depicted for a clipped $(A)$ and a control embryo $(B)$. The end-systolic points (open circles) and end-diastolic points (solid circles) were determined and were used to assess the ESPVR and EDPVR. From these relations, the $\mathrm{E}_{\mathrm{ES}}$ and the $\mathrm{E}_{\mathrm{ED}}$ were obtained, which are defined by the slopes of both relations, respectively. 
Table 1. Hemodynamic parameters derived from baseline pressure-volume loops at stage 21

\begin{tabular}{lcc}
\hline & Clip $(n=11)$ & Control $(n=10)$ \\
\hline $\mathrm{HR}(\mathrm{bpm})$ & $131 \pm 2$ & $140 \pm 5$ \\
$\mathrm{ESP}(\mathrm{mm} \mathrm{Hg})$ & $2.20 \pm 0.07$ & $2.14 \pm 0.13$ \\
$\mathrm{EDP}(\mathrm{mm} \mathrm{Hg})$ & $0.84 \pm 0.05$ & $0.71 \pm 0.06$ \\
$\mathrm{dP}_{\mathrm{dt}_{\mathrm{MAX}}(\mathrm{mm} \mathrm{Hg} / \mathrm{s})}$ & $41.3 \pm 1.8$ & $37.8 \pm 2.6$ \\
$\mathrm{dP}_{\mathrm{dt}}{ }_{\mathrm{MIN}}(\mathrm{mm} \mathrm{Hg} / \mathrm{s})$ & $-43.2 \pm 2.7$ & $-40.0 \pm 3.3$ \\
$\mathrm{ESV}(\mu \mathrm{L})$ & $0.20 \pm 0.02$ & $0.20 \pm 0.02$ \\
$\mathrm{EDV}(\mu \mathrm{L})$ & $0.41 \pm 0.02$ & $0.41 \pm 0.02$ \\
$\mathrm{SV}(\mu \mathrm{L})$ & $0.25 \pm 0.02$ & $0.24 \pm 0.01$ \\
$\mathrm{CO}(\mu \mathrm{L} / \mathrm{min})$ & $32.2 \pm 2.3$ & $32.6 \pm 1.3$ \\
$\mathrm{EF}(\%)$ & $59.3 \pm 3.0$ & $57.7 \pm 2.5$ \\
$\mathrm{SW}(\mu \mathrm{L} \cdot \mathrm{mm} \mathrm{Hg})$ & $0.44 \pm 0.03$ & $0.42 \pm 0.03$ \\
$\mathrm{PFR}(\mu \mathrm{L} / \mathrm{s})$ & $3.17 \pm 0.36$ & $3.55 \pm 0.40$ \\
PER $(\mu \mathrm{L} / \mathrm{s})$ & $-4.33 \pm 0.39$ & $-4.11 \pm 0.42$ \\
Tau $(\mathrm{ms})$ & $31.41 \pm 3.16$ & $39.02 \pm 6.20$ \\
PHT $(\mathrm{ms})$ & $22.67 \pm 1.66$ & $25.43 \pm 4.54$ \\
\hline
\end{tabular}

Values are mean \pm SEM. None of the parameters were significantly different between clipped and control embryos. $\mathrm{dP}_{\mathrm{dt}} \mathrm{MIN}_{\mathrm{N}}$, PER, Tau, and PHT were not normally distributed. Consequently, $t$ tests were performed after log transformation (using absolute values for $\mathrm{dP} / \mathrm{dt}_{\mathrm{MIN}}$ and PER) as described in "Methods."

contractility, whereas the EDPVR was steeper, indicating increased $\mathrm{E}_{\mathrm{ED}}$ (reduced compliance). The summarized results are given in Table 2 and show a significantly reduced contractility $(p=0.037)$ in the clipped embryos, indicated by a $52 \%$ reduction in $\mathrm{E}_{\mathrm{ES}}$. $\mathrm{E}_{\mathrm{ED}}$ tended to be increased in the clipped embryos as indicated by the $64 \%$ increase in $\mathrm{E}_{\mathrm{ED}}$, however, the difference did not reach statistical significance $(p=0.103)$.

Ventricular wall volume was obtained after application of 2 $\mathrm{M} \mathrm{NaCl}$ and was not significantly different between the clipped and control embryos $(0.195 \pm 0.013 \mu \mathrm{L}$ versus $0.181 \pm 0.017$ $\mu \mathrm{L}, \mathrm{NS})$.

\section{DISCUSSION}

The venous clip model is an intervention model for the chick embryo, in which permanent obstruction of the right lateral vitelline vein temporarily reduces the mechanical load on the embryonic myocardium and induces a spectrum of outflow tract anomalies, e.g. double outlet right ventricle, dextroposition of the aorta, and ventricular septal defects. The same types of anomalies can be present in humans. There is an intricate relationship between hemodynamics and cardiac morphogenesis. Pressure-volume loop analysis enables determination of $\mathrm{E}_{\mathrm{ES}}$ and $\mathrm{E}_{\mathrm{ED}}$ of the developing ventricle. These parameters represent intrinsic myocardial properties and are relatively

Table 2. End-systolic ventricular elastance $\left(E_{E S}\right)$, end-diastolic ventricular stiffness $\left(E_{E D}\right)$, and $E S V$ at $P=2 \mathrm{~mm} \mathrm{Hg}$ obtained from pressure-volume relations

\begin{tabular}{lcc}
\hline & Clip $(n=11)$ & Control $(n=10)$ \\
\hline $\mathrm{E}_{\mathrm{ES}}(\mathrm{mm} \mathrm{Hg} / \mu \mathrm{L})$ & $5.68 \pm 0.85^{*}$ & $11.76 \pm 2.70$ \\
$\mathrm{E}_{\mathrm{ED}}(\mathrm{mm} \mathrm{Hg} / \mu \mathrm{L})$ & $2.74 \pm 0.56$ & $1.67 \pm 0.21$ \\
$\mathrm{ESV}_{2}(\mu \mathrm{L})$ & $0.16 \pm 0.02$ & $0.18 \pm 0.01$ \\
\hline
\end{tabular}

Values are means \pm SEM. $\mathrm{E}_{\mathrm{ES}}, \mathrm{E}_{\mathrm{ED}}$, and $\mathrm{ESV}_{2}$ demonstrated inhomogeneity of variances. Therefore, a $\log (\ln )$ transformation was performed prior to performing $t$ tests.

$* p<0.05$. independent of loading conditions. The use of pressure-volume loop analysis in early stages of development could contribute to a better understanding of the relationship between cardiac function and morphogenesis during normal and abnormal cardiac development.

The cardiac cycle consists of four distinct phases: the isovolumic contraction phase, the ejection phase, the isovolumic relaxation phase, and the diastolic filling phase. Parameters that reflect the first two phases are $\mathrm{dP} / \mathrm{dt}_{\mathrm{MAX}}$ (isovolumic contraction phase), and $\mathrm{CO}, \mathrm{EF}, \mathrm{SW}$, and $\mathrm{E}_{\mathrm{ES}}$ (ejection phase). From these parameters, only the $\mathrm{E}_{\mathrm{ES}}$ was significantly changed in the clipped embryos. This may reflect the high sensitivity of this load-independent parameter of contractility compared with the other parameters that are also changed by alterations in preand afterload. The reduced $\mathrm{E}_{\mathrm{ES}}$ indicating reduced contractile state can be explained by observations of Hogers et al. (25). They observed less developed ventricles after venous clipping and a delay in cardiac looping. They did not observe normal embryos between stage 19 and 23 after clipping. All embryos displayed cardiovascular malformations. They found that stages 22-24 are critical for survival. A mortality rate between 10 and $22 \%$ is described. At later stages of development, approximately $64 \%$ of the embryos displayed cardiac malformations (4). Normally, myocardial contraction force increases with development $(5,26-29)$. After clipping, ventricular contractility development may lag behind as a consequence of impaired cardiac development. Mechanical load regulates ventricular growth $(30,31)$. Venous obstruction temporarily reduces mechanical load on the myocardium and thus probably slows down the ventricular growth and differentiation process (3). During normal development, the ventricular wall volume will increase in size. Since a delay in ventricular development was observed by Hogers et al. (25), we would have expected wall volume to be smaller in the clipped embryos. However, after applying $2 \mathrm{M} \mathrm{NaCl}$ to achieve maximum ventricular contraction, ventricular wall volume was similar between the groups. Potentially, the reduced contractility in the clipped embryos limits their maximal contracture leading to an overestimation in wall volume.

The third phase of the cardiac cycle, the active phase of relaxation represented by Tau, reflects the energy-consuming process of relaxation. In the mature heart, ventricular relaxation rate is an important determinant of the early diastolic pressure-volume relation (32). There were no significant differences in the active phase of relaxation between the two groups.

The end-diastolic pressure-volume relationship reflects passive relaxation properties of the ventricle, which contribute to the passive relaxation phase during diastolic filling. The relation was quantified by its slope, the $\mathrm{E}_{\mathrm{ED}}$, which was $64 \%$ higher in the clipped group. Although this was not a significant difference, this finding suggests reduced compliance after clipping. Myocardial compliance increases with development $(9,28,33)$. A higher $\mathrm{E}_{\mathrm{ED}}$ after venous obstruction may result from impaired cardiac development after clipping. Changes in structure of the ventricular wall may affect the stiffness of the ventricle, especially the addition and organization of myofibrils may limit cell distensibility (34). Normally, myofibrils become 
more organized with development and assume a more orderly arrangement, presumably parallel to stress lines (5). After venous obstruction this could be impaired. Next to the organization of myofibrils, titin is also important for passive stiffness (35). Titin contains passive, spring-like properties that help restore stretched muscle to its resting length (36). Diastolic pressure results from the tension that develops when passive myocardium is stretched beyond its slack length. In dogs with tachycardia-induced dilated cardiomyopathy, it has been demonstrated that titin isoform ratio changes cause an increase in myocardial stiffness (37). We speculate that titin isoform expression ratios may have been changed by clipping, causing increased passive stiffness.

Changes in diastolic function may be a critical regulatory pathway during cardiac morphogenesis (34). Recently Schroder et al. (31) found an increase in microtubule density and $\beta$-tubulin protein after reduced mechanical load in the stage 27 embryonic heart after left atrial ligation at stage 21. They observed increased myocardial stiffness in response to reduced mechanical load. They hypothesized that because microtubules mediate the transport of materials necessary for the production of new cellular structures, they may also play a role in mediating the adaptive response of the developing myocardium to altered mechanical load. Because, in the venous clip model, mechanical load is reduced, it could be possible that changes in microtubule density and organization caused the tendency of increased $\mathrm{E}_{\mathrm{ED}}$ in the clipped embryos. A decrease in workload may affect the number of contractile units or their function within the myocyte. Recently, we observed a reduction in the diastolic passive-filling component of ventricular filling in favor of the active component in clipped embryos at stage 24 (38). We speculate that a stiffer ventricular wall causes this decrease in passive filling, which is compensated by the active contraction of the atrium. It seems that at stage 21 the ventricle is just starting to become stiffer.

Study limitations. There are several limitations to the present study. Like Tobita and Keller $(29,39)$, we assumed that the embryonic ventricle is a thick-walled ellipsoidal shell with a fixed ratio of semiminor and semimajor axis diameter during systole. This could lead to error in the calculation of ventricular wall volume and cavity volume.

As demonstrated by our study in which the calculation of ventricular wall volume and assessing cavity volume was compared with volumes obtained by Cavalieri's method, there is a overestimation of ventricular wall volume by $8.9 \%$. In theory, this means that cavity volumes are underestimated by about $0.016-0.017 \mu \mathrm{L}$. Inasmuch as this represents, even for the smallest volumes (mean ESV was $0.20 \mu \mathrm{L}$ ), only about $8.7 \%$ of cavity volume, we chose not to correct for this factor. Furthermore, because this would cause a rightward shift of the entire pressure-volume loops, it has no consequences for the slopes of the pressure-volume relations.

We considered trabecular spaces to be part of the wall. These spaces do not close completely after application of $2 \mathrm{M}$ $\mathrm{NaCl}$, but for the calculation of ventricular volume this is not very important. These spaces are present during both diastole and systole. This results in a slight overestimation of total volumes throughout the cardiac cycle. Therefore, when wall volume is subtracted from the total volumes, this overestimation is cancelled out. Any remaining trabecular space volume can be considered dead volume, as this blood does not contribute to $\mathrm{CO}$.

Because we only obtained pressure and volume data before and after hemorrhage and not during this preload reduction, we chose linear relationships to describe the ESPVR and EDPVR. This may have influenced our results, but, because the pressure-volume relations were based on only two conditions (rather than on data acquired continuously during preload reduction), there was no justification for applying a nonlinear fit. Possible nonlinearities might have been detected when the ESPVR had been determined over a wider pressure range. However, systolic pressure was similar in both groups, thus comparison of $\mathrm{E}_{\mathrm{ES}}$ between groups is unlikely to be importantly affected by a pressure-dependent nonlinearity.

There were distinct differences in variances of the $\mathrm{E}_{\mathrm{ES}}$ and $\mathrm{E}_{\mathrm{ED}}$ between the clipped and control embryos before log (ln) transformation. Several factors may be involved. The chick embryos are not genetically identical, this could cause biologic variability. Also, the clipping experiment could have been partially successful, which could affect the outcome. There could have been some influence of the extent of hemorrhage. In theory, loading conditions do not influence the ESPVR or the EDPVR. However, in the small chick embryo this might be different. Also the position of the embryo and/or micropipette could have been changed during the incision of a fourth order vitelline vein. This could have caused some variation as well. Furthermore, temperature differences could have influenced the measurements as time to insert the micropipette into the ventricle varied between experiments. However, whereas these effects may increase within-group variability, they would not be expected to systematically affect the differences between the groups.

Because of the necessity to end the experiments with the use of $2 \mathrm{M} \mathrm{NaCl}$, it was impossible to obtain good histologic examinations of the measured embryonic hearts. Therefore, we compared our results with scanning electron microscope and histologic findings of Hogers et al. (25), who developed the venous clip model. Any existing discrepancies in morphologic findings between our data and those of Hogers et al. (25) could, therefore, not be detected.

In summary, development of ventricular function in the embryonic heart is affected by mechanical load. Pressurevolume loop analysis shows that, $1 \mathrm{~d}$ after venous obstruction, the clipped embryo has a reduced contractility compared with stage-matched control embryo's. The morphologic correlates of these findings require further study. Pressure-volume analysis may be applied in the chick embryo and is a sensitive technique for detecting subtle alterations in ventricular function.

\section{REFERENCES}

1. Clark EB, Hu N 1982 Developmental hemodynamic changes in the chick embryo from stage 18 to 27 . Circ Res 51:810-815

2. Nakazawa M, Miyagawa S, Ohno T, Miura S, Takao A 1988 Developmental hemodynamic changes in rat embryos at 11 to 15 days of gestation: normal data of blood pressure and the effect of caffeine compared to data from chick embryo. Pediatr Res 23:200-205 
3. Stekelenburg De Vos S, Ursem NT, Hop WC, Wladimiroff JW, Gittenberger-de Groot AC, Poelmann RE 2003 Acutely altered hemodynamics following venous obstruction in the early chick embryo. J Exp Biol 206:1051-1057

4. Hogers B, DeRuiter MC, Gittenberger-de Groot AC, Poelmann RE 1997 Unilateral vitelline vein ligation alters intracardiac blood flow patterns and morphogenesis in the chick embryo. Circ Res 80:473-481

5. Clark EB, Hu N, Dummett JL, Vandekieft GK, Olson C, Tomanek R 1986 Ventric ular function and morphology in chick embryo from stages 18 to 29. Am J Physiol 250:H407-H413

6. Hu N, Clark EB 1989 Hemodynamics of the stage 12 to stage 29 chick embryo. Circ Res 65:1665-1670

7. Broekhuizen ML, Mast F, Struijk PC, van der Bie W, Mulder PG, Gittenberger-de Groot AC, Wladimiroff JW 1993 Hemodynamic parameters of stage 20 to stage 35 chick embryo. Pediatr Res 34:44-46

8. Hamburger V, Hamilton HL 1951 A series of normal stages in the development of the chick embryo. J Morphol 88:49-92

9. Keller BB, Tinney JP, Hu N 1994 Embryonic ventricular diastolic and systolic pressure-volume relations. Cardiol Young 4:19-27

10. Kass DA, Yamazaki T, Burkhoff D, Maughan WL, Sagawa K 1986 Determination of left ventricular end-systolic pressure-volume relationships by the conductance (volume) catheter technique. Circulation 73:586-595

11. Teitel DF, Klautz R, Steendijk P, van der Velde ET, van Bel F, Baan J 1991 The end-systolic pressure-volume relationship in the newborn lamb: effects of loading and inotropic interventions. Pediatr Res 29:473-482

12. Georgakopoulos D, Mitzner WA, Chen CH, Byrne BJ, Millar HD, Hare JM, Kass DA 1998 In vivo murine left ventricular pressure-volume relations by miniaturized conductance micromanometry. Am J Physiol 274:H1416-H1422

13. Lewinsky RM, Szwarc RS, Benson LN, Ritchie JW 1993 The effects of hypoxic acidemia on left ventricular end-systolic elastance in fetal sheep. Pediatr Res 34:38-43

14. Weil SR, Russo PA, Heckman JL, Balsara RK, Pasiecki V, Dunn JM 1993 Pressurevolume relationship of the fetal lamb heart. Ann Thorac Surg 55:470-475

15. Keller BB, Yoshigi M, Tinney JP 1997 Ventricular-vascular uncoupling by acute conotruncal occlusion in the stage 21 chick embryo. Am J Physiol 273:H2861-H2866

16. Hove JR, Koster RW, Forouhar AS, Acevedo-Bolton G, Fraser SE, Gharib M 2003 Intracardiac fluid forces are an essential epigenetic factor for embryonic cardiogenesis. Nature 421:172-177

17. Heineman FW, Grayson J 1985 Transmural distribution of intramyocardial pressure measured by micropipette technique. Am J Physiol 249:H1216-H1223

18. Gundersen HJ, Jensen EB 1987 The efficiency of systematic sampling in stereology and its prediction. J Microsc 147:229-263

19. Sagawa K, Maughan L, Suga H, Sunagawa K 1988 Cardiac contraction and the pressure-volume relationship. Oxford University Press, New York, pp 36-39

20. Kono A, Maughan WL, Sunagawa K, Hamilton K, Sagawa K, Weisfeldt ML 1984

The use of left ventricular end-ejection pressure and peak pressure in the estimation of the end-systolic pressure-volume relationship. Circulation 70:1057-1065
21. Leeuwenburgh BP, Steendijk P, Helbing WA, Baan J 2002 Indexes of diastolic RV function: load dependence and changes after chronic RV pressure overload in lambs. Am J Physiol Heart Circ Physiol 282:H1350-H1358

22. Steendijk P, Baan J Jr, Van der Velde ET, Baan J 1998 Effects of critical coronary stenosis on global systolic left ventricular function quantified by pressure-volume relations during dobutamine stress in the canine heart. J Am Coll Cardiol 32:816-826

23. Pak PH, Maughan L, Baughman KL, Kass DA 1996 Marked discordance between dynamic and passive diastolic pressure-volume relations in idiopathic hypertrophic cardiomyopathy. Circulation 94:52-60

24. Bland JM, Altman DG 1996 Transforming data. BMJ 312:770

25. Hogers B, Gittenberger-de Groot AC, DeRuiter MC, Mentink MM, Poelmann RE 1998 Cardiac inflow malformations are more lethal and precede cardiac outflow malformations. Chick embryonic venous clip model. In: Hogers B (ed) The Role of Blood Flow in Normal and Abnormal Heart Development. Ponsen \& Looijen BV, Wageningen, The Netherlands, pp 79-100

26. Nakanishi T, Seguchi M, Takao A 1988 Development of the myocardial contractile system. Experientia 44:936-944

27. Godt RE, Fogaca RT, Nosek TM 1991 Changes in force and calcium sensitivity in the developing avian heart. Can J Physiol Pharmacol 69:1692-1697

28. Anderson PA 1996 The heart and development. Semin Perinatol 20:482-509

29. Tobita K, Keller BB 2000 Maturation of end-systolic stress-strain relations in chick embryonic myocardium. Am J Physiol Heart Circ Physiol 279:H216-H224

30. Clark EB, Hu N, Frommelt P, Vandekieft GK, Dummett JL, Tomanek RJ 1989 Effect of increased pressure on ventricular growth in stage 21 chick embryos. Am J Physiol 257:H55-H61

31. Schroder EA, Tobita K, Tinney JP, Foldes JK, Keller BB 2002 Microtubule involvement in the adaptation to altered mechanical load in developing chick myocardium. Circ Res 91:353-359

32. Gilbert JC, Glantz SA 1989 Determinants of left ventricular filling and of the diastolic pressure-volume relation. Circ Res 64:827-852

33. Friedman WF 1972 The intrinsic physiologic properties of the developing heart. Prog Cardiovasc Dis 15:87-111

34. Hu N, Connuck DM, Keller BB, Clark EB 1991 Diastolic filling characteristics in the stage 12 to 27 chick embryo ventricle. Pediatr Res 29:334-337

35. Granzier H, Labeit S 2002 Cardiac titin: an adjustable multi-functional spring. J Physiol 541:335-342

36. Epstein ND, Davis JS 2003 Sensing stretch is fundamental. Cell 112:147-150

37. Wu Y, Bell SP, Trombitas K, Witt CC, Labeit S, LeWinter MM, Granzier H 2002 Changes in titin isoform expression in pacing-induced cardiac failure give rise to increased passive muscle stiffness. Circulation 106:1384-1389

38. Ursem NT, Stekelenburg-de Vos S, Wladimiroff JW, Poelmann RE, Gittenberger-de Groot AC, Hu N, Clark EB 2004 Ventricular diastolic characteristics in stage-24 chick embryos after extra-embryonic venous obstruction. J Exp Biol 207:1487-1490

39. Tobita K, Keller BB 1999 End-systolic myocardial stiffness is a load-independent index of contractility in stage 24 chick embryonic heart. Am J Physiol 276:H2102_ 2108 